\title{
Effects of Lift Velocity on Muscle Activation During Leg Extension
}

\author{
Brian M. Hatzel*, Stephen C. Glass, Scott Johnson and Heather Sjoquist
}

Human Performance Laboratory, Grand Valley State University, Allendale, MI, USA

\begin{abstract}
It is not known if manipulating velocity within a prescribed resistance training mode will improve muscle activation. Muscle activations of the Rectus Femoris (RF), Vastus Lateralis (VL), Vastus Medialis (VM) and Bicep Femoris (BF) were examined during a leg extension exercise at 3 different velocities on 15 subjects (10men, 5 female, Age $=21.5 \pm 1.8 \mathrm{yrs}$, Height $=171.2 \pm 12.5 \mathrm{~cm}$, Mass $=75.5 \pm 16.3 \mathrm{~kg}$ ). Trials of 1 set of 10 repetitions at $60 \%$ of $1 \mathrm{RM}$, were performed at 15, 30 and $60 \%$ s. Bipolar surface electrodes were placed over the BF, RF, VL, and VM. Micro-switches were utilized to identify the concentric $(\mathrm{CON})$ and eccentric (ECC) phases of the lift. Data were sampled at $1024 \mathrm{~Hz}$, filtered, rectified and the mean, integrated EMG calculated. One 2 x 4 x 3 (action x muscle x velocity) ANOVA with bonferonni adjustment was run and significance was followed by Tukey HSD post hoc analysis. Results indicated significantly greater activation of the VL, RF and VM for ECC extension at $60 \%$ s compared to $15 \%$ s. While $60 \%$ s was also greater than $30 \%$ for the VL and VM during ECC. While comparing muscle action, CON VL, VM and RF were greater than ECC at $30 \% \mathrm{sec}$, meanwhile VM CON was also greater at $15 \%$ sec. No differences in muscle activation at any velocity or muscle action for BF were identified. We conclude that muscle recruitment while training with a $60 \% 1 \mathrm{RM}$ load is maximized at a velocity of $60 \%$ s during ECC activity and 15 or $30 \%$ sec during CON.
\end{abstract}

Keywords: EMG, lift speed, muscle recruitment, training velocity.

\section{INTRODUCTION}

In the health and fitness setting, the goal of strength training programs is often to establish appropriate resistance training loads in an attempt to maximize outcomes for strength and performance. Traditionally, these programs have focused on theories based on the number of repetitions, numbers of sets and the amount of weight to be lifted in order to maximize muscular output [1-7]. Proper instructions in regards to lifting technique, in combination with adequate volume (sets, reps) often are given only during initial consultations.

Another aspect of the exercise sometimes identified, is the velocity of the lift. It is well documented that the velocity of movement of a load is inversely related to the load, so the heaviest loads elicit the slowest lift velocity [1]. Conversely, the lighter loads can be moved at much higher velocities. For novice to intermediate weight lifters it is recommended that training intensity be $60-70 \%$ of 1 RM with $8-12$ repetitions $[1,8,9]$. At these loads a range of velocities exist where the individual can complete the repetitions while maintaining proper form. However it is not clear if muscle activation varies within these velocities. Traditional instructions have involved a slow concentric phase followed by a brief pause then concluded with an even slower eccentric phase. Anecdotally, it has been thought that this slow training maximizes strength gain, and some authors have advocated for super slow training $[10,11]$. Within these load ranges, some variation in speed has been examined. At

*Address correspondence to this author at the Grand Valley State University, 1 Campus Dr. MAK B2-218, Allendale, MI 49401, USA; Tel: 616-331-8538; Fax: 616-331-8870; E-mail: hatzelb@gvsu.edu one end of the speed spectrum, slow training has not shown itself to be as effective as faster movement for strength development. Hatfield et al. [12] compared slower velocity training (10s concentric, $5 \mathrm{~s}$ eccentric) at 60 and $80 \%$ of 1 $\mathrm{RM}$ to a self-selected lift cadence. They found that subjects who completed significantly lower training volume during slower training, indicated a higher effort rating, and had a reduced power output compared to the faster self-selected lift speed.

Much of the research examining slower velocity resistance training has suggested poor outcomes in respect to strength gains, muscle recruitment, metabolic cost and lifting volume [1]. Many investigations examining lifting velocities have considered those that are either much slower than conventional practices [13] or much faster [2, 14, 15]. In addition, most studies have used isokinetic or hydraulic exercise modes, rather than isotonic [16]. It is not known to what degree minor variations in lift velocity affect the amount of muscle activation experienced during a lift. Authors [14, 17-20] have identified that more hypertrophy and / or strength gains have come from training protocols involving higher ECC velocities. However, it should be noted that the velocities examined were higher than the present investigation. Concentric resistance training recruits more muscle at low velocities, with force production dropping as the velocity increases. Conversely, ECC contractions are greatest at higher velocities. These changes are seen across a large range of contraction velocities. It is not known whether there are changes in muscle activation patterns across a more narrow range of speeds, and one that utilizes a load conducive to strength gain. If subtle variations in velocity do not result in activation changes, conventional speed model exercise prescriptions would be adequate. 
However, if slight variations $\left(15,30\right.$ and $\left.60^{\circ} / \mathrm{sec}\right)$ in velocity do result in activation differences, exercise prescriptions should reflect those benefits.

The purpose of this investigation was to examine muscle activation during a leg extension exercise completed at three lift velocities. The highest velocity that could be completed while maintaining technique was $60^{\circ} / \mathrm{sec}$, while two slower $\left(15,30^{\circ} / \mathrm{sec}\right)$ velocities were chosen to represent a practical range of lifting velocities that may be encountered in a typical fitness setting.

\section{MATERIALS AND METHODOLOGY}

Past research has indicated that strength development occurs when using loads ranging from $60 \%$ - 95\% 1RM [1]. The present investigation examined the use of a load suitable for strength development while varying the velocity of the lift and assessing muscle activation. Pilot testing assisted the authors with determining the lifting velocities that subjects would be able to complete with proper form, while the slowest velocity was chosen to create a condition similar to slower training [21].

Fifteen subjects $(10 \mathrm{men}, 5$ female, Age $=21.5 \pm 1.8 \mathrm{y}$, Height $=171.2 \pm 12.5 \mathrm{~cm}$, Mass $=75.5 \pm 16.3 \mathrm{~kg}$ ) were recruited for this investigation. Subjects were familiar with strength training, and had been actively lifting within the past six months. Subjects provided signed consent in accordance with the Institutional Review Board, who approved the study. All subjects completed an initial orientation day, where they were provided a familiarization with the activity, machine and completed an estimation of one repetition maximum (1RM). Subjects were positioned according to manufacturer recommendations for the variable resistance, leg extension exercise (Badger Magnum Selecterized Equipment) and instructed as to the proper form. An estimated 1RM for leg extension was assessed using the equation of Bryzycki [22]. Cadence was practiced at $15^{\circ} / \mathrm{sec}(6 \mathrm{~s}$ concentric, 6s eccentric), $30 \% / \mathrm{sec}(3 \mathrm{~s}$ concentric, $3 \mathrm{~s}$ eccentric) and $60^{\circ} / \mathrm{sec}(1.5 \mathrm{~s}$ concentric, $1.5 \mathrm{~s}$ eccentric). A metronome and a micro-switch at each end of the range of motion assisted the subjects' performance at the proper cadence and within a standardized range of motion.

Subjects reported to the Human Performance laboratory on a second occasion for trial testing. The dominant leg, as determined by handedness of the subject, was used for electrode placement. The skin was prepped for electrode placement by shaving the designated areas to remove hair and abraded using a coarse pad and rubbed clean with rubbing alcohol and a towel. These procedures were followed until the skin impedance was found to be less than $10,000 \mathrm{ohms}$ using a standard ohmmeter [23].

The Biopac ${ }^{\circledR}$ Tel-100 EMG system (Goleta, CA) was used to measure muscle electrical activity and record the data from each subject. The EMG data were analyzed using Acqknowledge ${ }^{\mathrm{TM}}$ software. Bipolar adhesive surface electrodes ( $\mathrm{Ag}-\mathrm{AgCl}, 2 \mathrm{~cm}$ inter electrode distance) were used over the muscle bellies of the involved muscles. The electrodes were placed parallel to the direction of the muscle fibers on the vastus medialis oblique (VM) vastus lateralis $(\mathrm{VL})$, biceps femoris $(\mathrm{BF})$ and rectus femoris (RF). The fibers of the VM run at approximately a 55-degree angle medial to the quadriceps tendon, and the electrode was placed $20 \%$ of the distance from the medial joint line of the knee to the anterior superior iliac spine. The fibers of the VL are at 12 to 15 -degrees lateral to the quadriceps tendon; the electrode was placed at the midpoint between the head of the greater trochanter and the lateral femoral epicondyle. Ground electrodes were placed on the patella and 6 to $8 \mathrm{~cm}$ from the inferior pole of the patella along the bony shaft of the anterior tibia. For the BF, electrodes were placed on the posterior thigh along a line of reference between the ischial tuberosity and the lateral popliteal surface directly over the gaster of the muscle. A ground electrode was placed on the head of the fibula. For the RF, electrodes were placed on the anterior aspect of the thigh midway between the anterior superior iliac spine and the patella. A ground electrode was placed on the tibial tuberosity.

Subjects completed one set of 10 repetitions at $60 \%$ of $1 \mathrm{RM}$, performed at 15,30 and $60^{\circ} / \mathrm{sec}$, representative of the range of lifting velocities. Trial order was counterbalanced, and subjects were given 5 minutes between each set to rest. Subjects were asked to match the cadence set by the metronome for each trial. When a subject was unable to match the cadence, the trial was suspended, the subject rested, and the trial repeated. Two separate channels on the Biopac were configured into the digital box to accept input from switches denoting the starting and ending range (90180 degrees) of motion, and were activated by the arm of the leg extension equipment.

Data were sampled at $1024 \mathrm{~Hz}$ (gain $=2,000$ ) and stored. Post-test filtering to smooth motion artifact was conducted using Acqknowledge Software (Microsoft Corp.). A highpass filter with a cutoff frequency of $30 \mathrm{~Hz}$ using 255 coefficients (Blackman -67) was used. Data were then rectified and integrated. The final IEMG values for each concentric and eccentric phase of the exercise were identified using the micro-switch data (Fig. 1). Samples were averaged across 5 repetitions (repetitions 3-7) in order to avoid initial errors in velocity as well as fatigue effects [23].

One $2 \times 4 \times 3$ (action $\times$ muscle $\mathrm{x}$ velocity) ANOVA was used; significance was followed by Tukey HSD post hoc analysis. Velocity effects on muscle activation were of interest, therefore gender effects were not considered in this analysis. Data were analyzed using the SPSS software14.0. (Chicago, IL). Significance was set at $p<.05$ and Bonferroni adjustments were made. Stability of IEMG data were analyzed by calculation of intra-class correlation (ICC) coefficients. In all cases, ICC values exceeded .90 (range .904 to .994$)$.

\section{RESULTS}

Significantly greater activation was identified in the VL $(\mathrm{p}=.001)$, RF (.003) and VM (.000) for ECC extension at $60 \% \mathrm{sec}$ compared to $15 \% \mathrm{sec}$ (Figs. 3-5). While $60^{\circ} / \mathrm{sec}$ was also greater than $30 \%$ sec for the VL (.023) and VM (.019) during ECC (Figs. 3, 5). While comparing muscle action, CON VL (.007), VM (.004) and RF (.025) were greater than their respective ECC at $30 \% \mathrm{sec}$, meanwhile VM CON (.001) was also greater at $15^{\circ} / \mathrm{sec}$ (Figs. 3-5). No differences in muscle activation at any velocity or muscle action for BF were identified (Fig. 2). 


\section{Subject 10 Leg Extension: 30 degrees/ sec}

Bicep
Femoris

jowoss

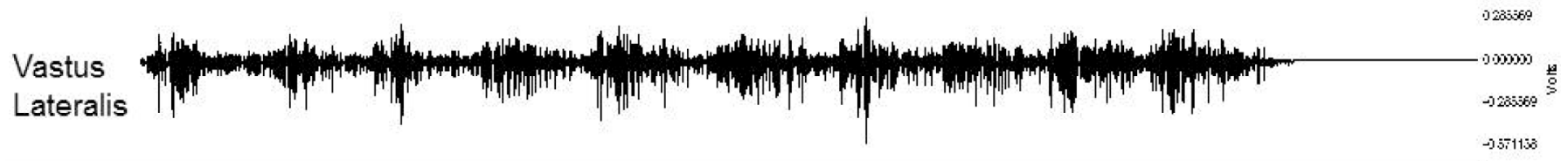

Rectus
Femoris

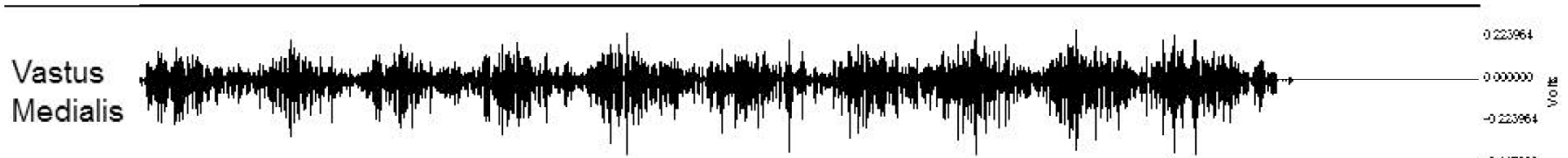

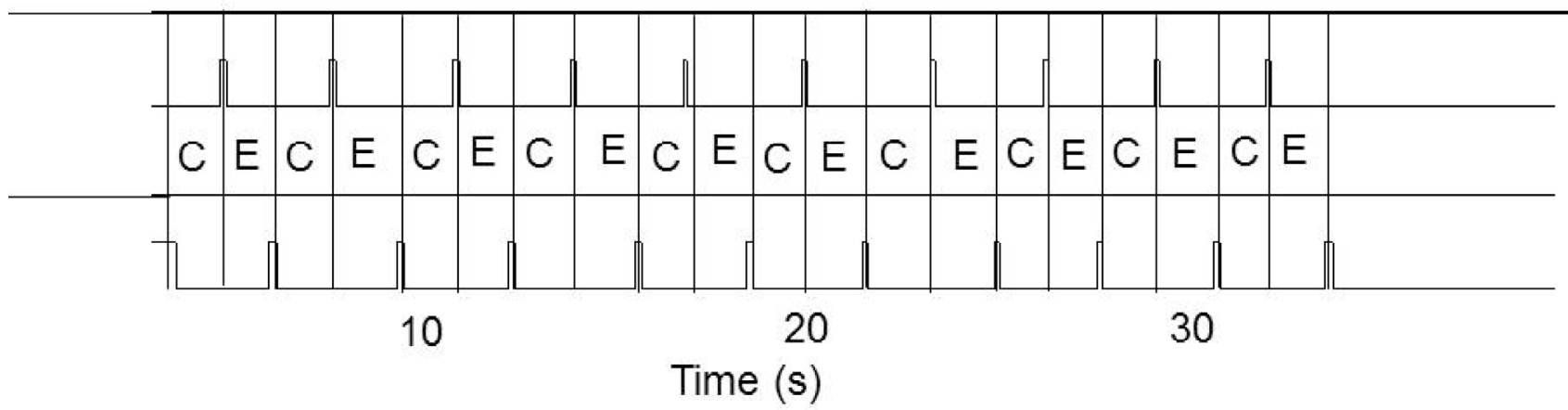

Fig. (1). Example of EMG sampling during leg extension trial: 30 degrees per second. C denotes Concentric and E denotes Eccentric. Contractions are divided by electronic position markers.

Biceps Femoris Recuitment Leg Extension Concentric and Eccentric

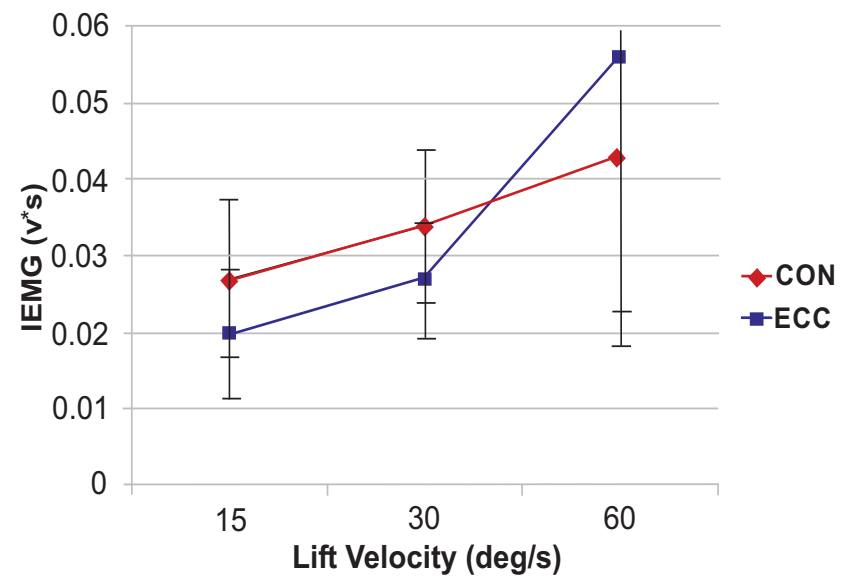

Fig. (2). Biceps Femoris activation during leg extension across lift velocities. No significant differences identified.

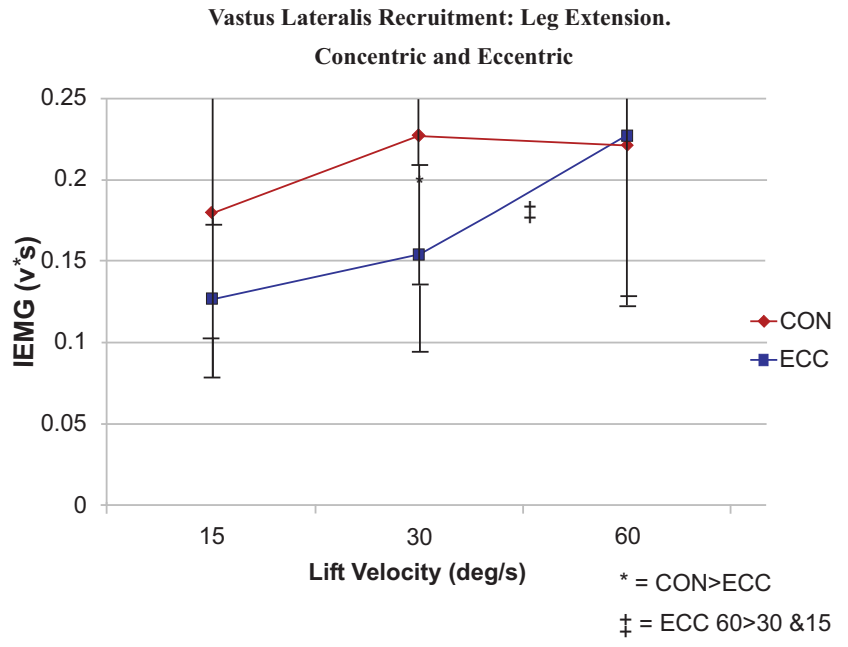

Fig. (3). Vastus Lateralis activation during leg extension across lift velocities. ECC $60 \%$ sec significantly greater than both 30 and 15 $\%$ sec. No differences during $\mathrm{CON}$ at any velocity. $\mathrm{CON}$ is greater than ECC at $30 \%$ sec. 


\section{DISCUSSION}

The major finding of this study is that ECC training at 60 $\% / \mathrm{sec}$ was responsible for greater peak muscle activation when compared to slower lifting velocities (15 and $30^{\circ} / \mathrm{sec}$ ). The present investigation is consistent with previous findings of greater muscle hypertrophy, strength and activation in response to faster ECC training [18-20]. An interesting aspect of the present project is that despite an only $45 \% \mathrm{sec}$ difference from the slowest $\left(15^{\circ} / \mathrm{sec}\right)$ to the fastest velocity $\left(60^{\circ} / \mathrm{sec}\right)$ that significant differences were identified in muscle activation. This is an important finding and has direct implications in the exercise prescription of recreational lifters. Farthing and Chilibeck [20] implemented an 8 week investigation looking at the effect of eccentric and concentric training at slow $(30 \% \mathrm{sec})$ and fast velocities $(180 \% \mathrm{sec})$. Eccentric training at the higher velocity showed a $13 \%$ increase in hypertrophy when compared to eccentric training at slower velocities $(7.8 \%)$.

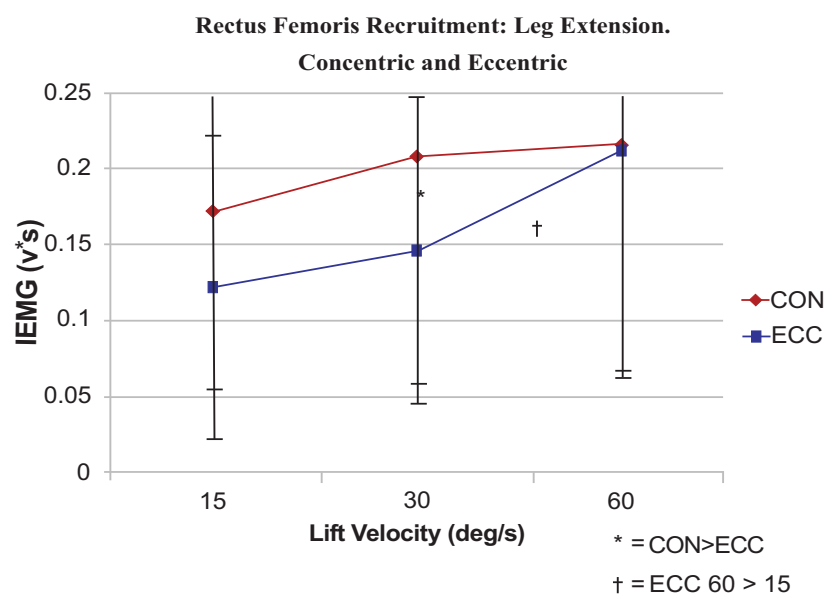

Fig. (4). Rectus Femoris activation during leg extension across lift velocities. ECC $60 \%$ sec significantly greater than $15 \% \mathrm{sec}$. CON is greater than ECC at $30 \%$ sec.

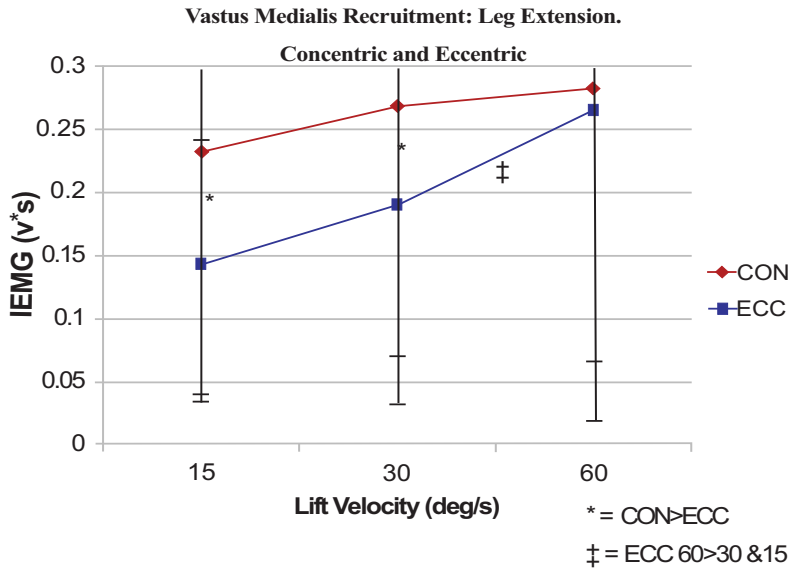

Fig. (5). Vastus Medialis activation during leg extension across lift velocities. ECC $60 \%$ sec significantly greater than both 30 and 15 $\%$ sec. CON is greater than ECC at 30 and $15 \%$ sec.

Some subscribe to the theory of super slow resistance training (10 sec CON, $4 \mathrm{sec}$ ECC). The theory is based on the thought that the tension within a muscle is related to the number of motor units firing and to the frequency with which impulses are conveyed to the motor neurons [24]. Using a slower speed is thought to require the activation of more muscle fibers and an increase in the frequency of firing in order to maintain a force necessary to lift a given workload [25]. Westcott [11] investigated on two occasions the effects of a slow training (10 sec CON, $4 \mathrm{sec}$ ECC) regimen in contrast to a regular training velocity (2 sec CON, 4 sec ECC) program [11]. A 12.0 and $10.9 \mathrm{~kg}$ increase in the slow velocity group were noted compared to an 8.0 and $7.1 \mathrm{~kg}$ increase in the regular training velocity group. These findings are disputed by that of Paddon - Jones et al. [26,27] and Farthing and Chilibeck et al. [20] who showed that eccentric training at higher velocities $\left(180^{\circ} / \mathrm{sec}\right)$ produced greater increases in strength than training at slower velocities $\left(30^{\circ} / \mathrm{sec}\right)$. Additionally, Neils and Udermann [14] examined the effects of an 8-week resistance training program of either a traditional resistance training program (2 sec CON, 4 sec ECC) or a super slow training program (10 $\sec$ CON, 5 sec ECC). They found greater increases in muscular power in the traditional program when examining the countermovement jump. They continued by stating that a super slow program is not an optimal method of training and the specificity of a short concentric contraction phase tends to favor explosive activities, which they evidenced by the $8.4 \%$ increase experienced in the traditional resistance training protocol. It should be noted that the investigation by Neils and Udermann [14] involved an 8 week training protocol and used resistance as their outcome measure, while the current investigation examined muscle activation through EMG.

It is difficult to identify a specific mechanism that is responsible for the disparities that exist in the findings of others investigating the relationship of velocity on muscle function. Investigations have been shown to vary by measurements (hypertrophy, strength, and activation), training regimen (frequency, duration) and by the specifics of velocity during each repetition. For instance some authors controlled the CON and ECC phases independently $[11,14]$ while others $[20,27]$ kept the velocity consistent throughout the task. Since force production varies by training velocity and contraction type, it is interesting that few studies exist that examine the specific mechanisms of such determinants $[18,20,28,29]$. As a function of the force - velocity relationship typically with concentric contractions force output decreases significantly with increasing contraction velocity [30-32]. Conversely, with eccentric contractions, force output increases with increasing contraction velocity $[32,33]$. Our findings support such a determinant. We noted at slower velocities $\left(15\right.$ and $\left.30^{\circ} / \mathrm{sec}\right)$ that $\mathrm{CON}$ activation was significantly greater than ECC activation within velocity. This provides further support that as velocity increases muscle activation during CON phase activation is attenuated.

The present investigation manipulated velocity by defining the CON and ECC phases as being the same in length. In all cases the CON phase equaled the timing of the ECC phase. Based on this model we were able to show that a significant increase in activation occurred at faster velocities eccentrically. Additionally, when comparing within each velocity we identified more activation at 15 and $30 \% \mathrm{sec}$ within the CON phase of the repetition. Across all findings it is clear that most activation occurs during faster ECC contractions and the CON activation patterns begin to taper off as velocity increases. Differences in activation are further separated as velocity increases [20]. Some authors have 
speculated that changes in muscle fiber type or increased ability to selectively recruit fast-twitch motor units may play a role in the higher activation noted at increased velocities [26]. Others have also stated that the same amount of work at a lower metabolic cost and perceived rate of exertion may be a result of ECC training [34-36]. Additionally, when it pertains to concentric contractions at slower velocities it has been reported that within the muscle fiber, the slower the rate at which the actin and myosin filaments slide past each other, the greater the number of links or cross-bridges that can be formed between them [25]. The more the crossbridges there exist per unit of time, the more tension is created and therefore at slow contraction velocities a higher number of cross-bridges can be formed leading to higher tension development.

Current strength and conditioning guidelines recommend resistance training at or above $60 \% 1 \mathrm{RM}$ to achieve increases in strength $[1,8]$. Meanwhile, it is important to identify the velocity by which participants may experience maximum muscular activation while maintaining a proper technique. Others have purported that higher velocity training increases muscle activation [5, 21]. However, these investigations included exercise techniques (Olympic lifts, ballistic training) that differ from isotonic training. Our findings indicate that muscle activation can be enhanced at modest loads by lifting at a slightly higher velocity during the ECC phase of the movement. The findings of greater activation ECC during faster movements and the growing difference between activation during slow and fast contraction velocities allows the current authors to postulate that increases in muscle activation will continue as functional performance occurs. Programs that include a functional velocity into resistance training may see additional benefits not noted in the traditional resistance training programs.

The present investigation found during ECC muscle activity that slightly increased velocity resistance training programs within a functional range of motion results in significantly greater muscle activation than at slower speeds. Strength and velocity are both important components of functional performance [1], and our data suggest that one can train at a load sufficient for strength gain, while also improving muscle activation, simply by increasing the speed of movement. It should be noted that this investigation did not examine the effect of slower speed training with higher resistance rather only looked at the effects on recruitment from varied velocities with a constant resistance.

\section{CONCLUSION}

While athletic populations typically train specifically for improving aspects of muscular strength, power and agility, recreational populations often do not employ extensive training regimens. Instead, they may only use a traditional resistance training session, exercising major muscle groups two or three days per week. Our data suggest that simply training at a slightly higher velocity of ECC with proper technique will significantly increase muscle activation. Training instructions could be modified to suggest that the client concentrically accelerates in a controlled manner while still maintaining a smooth motion, and eccentrically lowers at a faster velocity which still can be controlled.

\section{CONFLICT OF INTEREST}

The authors confirm that this article content has no conflict of interest.

\section{ACKNOWLEDGEMENTS}

Declared none.

\section{REFERENCES}

[1] American College of Sports Medicine. Progression models in resistance training for healthy adults. Med Sci Sports Exerc 2009; 41(3): 687-708.

[2] Drinkwater EJ, Lawton TW, Lindsell RP, et al. Training leading to repetition failure enhances bench press strength gains in elite junior athletes. J Strength Cond Res 2005; 19(2): 382-8.

[3] Fatouros IG, Kambas A, Katrabasas I, et al. Strength training and detraining effects on muscular strength, anaerobic power, and mobility of inactive older men are intensity dependent. Br J Sports Med 2005; 39(10): 776-80.

[4] Fielding RA, LeBrasseur NK, Cuoco A, et al. High-velocity resistance training increases skeletal muscle peak power in older women. J Am Geriatr Soc 2002; 50(4): 655-62.

[5] Kawamori N, Haff GG. The optimal training load for the development of muscular power. J Strength Cond Res 2004; 18(3): 675-84.

[6] Moss BM, Refsnes PE, Abildgaard A, et al. Effects of maximal effort strength training with different loads on dynamic strength, cross sectional area, load-power and load-velocity relationships. Eur J Appl Physiol 1997; 75: 193-9.

[7] Petrella JK, Kim JS, Tuggle SC, et al. Contributions of force and velocity to improved power with progressive resistance training in young and older adults. Eur J Appl Physiol 2007; 99(4): 343-51.

[8] American College of Sports Medicine. ACSM's guidelines for exercise testing and prescription. $8^{\text {th }}$ ed. USA: Lippincott Williams and Wilkins 2009.

[9] Campos GE, Luecke TJ, Wendeln HK, et al. Muscular adaptations in response to three different resistance-training regimens: specificity of repetition maximum training zones. Eur J Appl Physiol 2002; 88(1-2): 50-60.

[10] Hutchins K. Super slow: the ultimate exercise protocol. $2^{\text {nd }}$ ed. Casselberry, FL: Media Support 1993.

[11] Westcott WL, Winett RA, Anderson ES, et al. Effects of regular and slow speed resistance training on muscle strength. J Sports Med Phys Fitness 2001; 41(2): 154-8.

[12] Hatfield DL, Kraemer WJ, Spiering BA, et al. The impact of velocity of movement on performance factors in resistance exercise. J. Strength Cond Res 2006; 20(4): 760-6.

[13] Morrissey MC, Harman EA, Frykman PN, et al. Early phase differential effects of slow and fast barbell squat training. Am J Sports Med 1998; 26(2): 221-30.

[14] Neils CM, Udermann BE, Brice GA, et al. Influence of contraction velocity in untrained individuals over the initial early phase of resistance training. J Strength Cond Res 2005; 19(4): 883-7.

[15] Sakamoto A, Sinclair PJ. Effect of movement velocity on the relationship between training load and the number of repetitions of bench press. J Strength Cond Res 2006; 20(3): 523-7.

[16] Pereira MI, Gomes PS. Movement velocity in resistance training. Sports Med 2003; 33(6): 427-38.

[17] Raj IS, Bird SR, Shield AJ. Aging and the force-velocity relationship of muscles. Exp Gerontol 2010; 45: 81-90.

[18] Hortobagyi T, Barrier J, Beard D, et al. Greater initial adaptations to submaximal muscle lengthening than maximal shortening. J Appl Phys 1996; 81(4): 1677-82.

[19] Shepston TN, Tang JE, Dallaire S, et al. Short-term high- vs lowvelocity isokinetic lengthening training results in greater hypertrophy of the elbow flexors in young men. J Appl Phys 2005; 98(5): 1768-76.

[20] Farthing JP, Chilibeck PD. The effect of eccentric and concentric training at different velocities on muscle hypertrophy. Eur J Appl Physiol 2003; 89(6): 578-86.

[21] Haff GG, Carlock JM, Hartman MH, et al. Force-time curve characteristics of dynamic and isometric muscle actions of elite women Olympic weightlifters. J Strength Cond Res 2005; 19(4): $741-8$. 
[22] Brzycki M. Strength testing: predicting a one-rep max from a repsto-fatigue. J Phys Educ Recreat Dance 1993; 64(1): 88-90.

[23] Basmajian JV, De Luca CJ. Muscles alive: their functions revealed by electromyography. $5^{\text {th }}$ ed. Baltimore, MD: Williams \& Wilkins 1985.

[24] Berger RA. Applied exercise physiology. Philadelphia, PA: Lea and Febiger 1982.

[25] Smith LK, Weiss EL, Lehmkuhl LD. Brunnstrom's clinical kinesiology. $5^{\text {th }}$ ed. Philadelphia, PA: F.A. Davis Company 1996.

[26] Paddon-Jones DM, Leveritt A, Lonergan P, Abernethy P. Adaptation to chronic eccentric exercise in humans: the influence of contraction velocity. Eur J Appl Physiol 2001; 85: 466-71.

[27] Munn J, Herbert RD, Hancock MJ, Gandevia SC. Resistance training for strength: effect of number of sets and contraction speed. Med Sci Sports Exerc 2005; 37(9): 1622-6.

[28] Seger JY, Arvidsson B, Thorstensson A. Specific effects of eccentric and concentric training on muscle strength and morphology in humans. Eur J Appl Physiol 1998; 79: 49-57.

[29] Higbie EJ, Cureton KJ, Warren GL, Prior BM. Effects of concentric and eccentric training on muscle strength, crosssectional area, and neural activation. J Appl Physiol 1996; 81: 2173-81.
[30] Wickiewicz TL, Roy RR, Powell PL, Perrine JJ, Edgerton VR. Muscle architecture and force-velocity relationships in humans. J Appl Physiol 1984; 57: 435-43.

[31] Sale DG, MacDougall JD, Always SE, Sutton JR. Voluntary strength and muscle characteristics in untrained men and women bodybuilders. J Appl Physiol 1987; 62: 1786-93.

[32] Hortobagyi T, Katch FI. Eccentric and concentric torque-velocity relationships during arm flexion and extension. Eur J Appl Physiol 1990; 60: 395-401.

[33] Westing HS, Creswell AG, Thorstensson A. Muscle activation during maximal voluntary eccentric and concentric knee extension Eur J Appl Physiol 1991; 62: 104-8.

[34] Meyer K, Steiner R, Lastayo P, et al. Eccentric exercise in coronary patients: central hemodynamic and metabolic responses. Med Sci Sports Exerc 2003; 35(7): 1076-82.

[35] Okamoto T, MasuharaM, Ikuta K. Cardiovascular responses induced during high-intensity eccentric and concentric isokinetic muscle contraction in healthy young adults. Clin Physiol Funct Imaging 2006; 20(5): 39-44.

[36] Overend TJ, Versteegh TH, Thompson E, et al. Cardiovascular stress associated with concentric and eccentric isokinetic exercise in young and older adults. J Gerontol A Biol Sci Med Sci 2000; 55(4): 177-82.

Received: July 2, 2012

(C) Hatzel et al.; Licensee Bentham Open.

This is an open access article licensed under the terms of the Creative Commons Attribution Non-Commercial License (http://creativecommons.org/licenses/by-nc/3.0/) which permits unrestricted, non-commercial use, distribution and reproduction in any medium, provided the work is properly cited. 